\title{
Improving dark energy constraints with high-redshift Type la supernovae from CANDELS and CLASH
}

\author{
Vincenzo Salzano ${ }^{1}$, Steven A. Rodney ${ }^{2}$, Irene Sendra ${ }^{1}$, Ruth Lazkoz ${ }^{1}$, Adam G. Riess ${ }^{2,3}$, Marc Postman ${ }^{3}$, \\ Tom Broadhurst ${ }^{1}$, and Dan Coe $^{3}$ \\ ${ }^{1}$ Fisika Teorikoaren eta Zientziaren Historia Saila, Zientzia eta Teknologia Fakultatea, Euskal Herriko Unibertsitatea UPV/EHU, \\ 644 Posta Kutxatila, 48080 Bilbao, Spain \\ e-mail: enzo.salzano@gmail.com \\ 2 Department of Physics and Astronomy, The Johns Hopkins University, Baltimore, MD 21218, USA \\ 3 Space Telescope Science Institute, Baltimore, MD 21218, USA
}

Received 19 April 2013 / Accepted 24 June 2013

\section{ABSTRACT}

\begin{abstract}
Aims. We investigated the degree of improvement in dark energy constraints that can be achieved by extending Type Ia supernova (SN Ia) samples to redshifts $z>1.5$ with the Hubble Space Telescope (HST), particularly in the ongoing Cosmic Assembly Near-infrared Deep Extragalactic Legacy Survey (CANDELS) and the Cluster Lensing and Supernova survey with Hubble (CLASH) multi-cycle treasury programs.

Methods. Using the popular Chevalier-Polarski-Linder (CPL) parametrization of the dark energy $w=w_{0}+w_{a}(1-a)$ we generated mock SN Ia samples that can be projected out to higher redshifts. The synthetic datasets thus generated were fitted to the CPL model, and we evaluated the improvements that a high- $z$ sample can add to improve the statistical and systematic uncertainties on cosmological parameters.

Results. In an optimistic but still very achievable scenario, we find that extending the HST sample beyond CANDELS+CLASH to reach a total of $28 \mathrm{SN}$ Ia at $z>1.0$ could improve the uncertainty in the $w_{\mathrm{a}}$ parameter $\sigma_{w_{\mathrm{a}}}$ by up to $21 \%$. The corresponding improvement in the figure of merit (FoM) would be as high as $28 \%$. Finally, we consider the use of high-redshift SN Ia samples to detect non-cosmological evolution in SN Ia luminosities with redshift, finding that these tests could be undertaken by future space-based infrared surveys using the James Webb Space Telescope (JWST).
\end{abstract}

Key words. dark energy - cosmology: observations - supernovae: general

\section{Introduction}

The seminal works of Riess et al. (1998) and Perlmutter et al. (1999) presented the first solid evidence for the present acceleration of the universe by extending the Hubble diagram of Type Ia supernovae (SN Ia) to redshifts $z>0.5$. The use of SN Ia as distance indicators has now become a key component of modern investigations. However, we still lack a clear picture about the nature and the fundamental properties of the so-called dark energy driving this acceleration. As a consequence, many observational and theoretical problems posed in the last 15 years have not yet been addressed satisfactorily. The consensus cosmological model, the $\Lambda$ cold dark matter ( $\Lambda$ CDM; Carroll et al. 1992; Sahni \& Starobinski 2000), based on the well-known cosmological constant $\Lambda$, provides a good fit to most of the data (Tegmark et al. 2004; Seljak et al. 2005; Sanchez et al. 2006; Komatsu et al. 2011). This model, however, is also affected by serious theoretical shortcomings (Liddle 1998). Many possible alternatives have been introduced by our imaginative community, including modifications of the theoretical framework that deviate from general relativity such as Chaplygin gas, Cardassian expansion, DGP brane-world models, $f(R)$ theories, etc. (Tsujikawa 2010; Dvali 2006; Carroll et al. 2005; Freese \& Lewis 2002; Gorini et al. 2003; Bento et al. 2002; Sahni et al. 2003; Maartens 2007; Sotiriou et al. 2010). Among observers, the most popular class of alternative models are the proposals for evolving dark energy. There is no strong theoretical reason for dark energy density to remain constant as the universe expands, and simple models for dark energy evolution can already be investigated with currently accessible cosmological probes (Weinberg et al. 2012).

Any fundamental component of the universe can be described, among other things, by its equation of state (EoS), defined as the ratio of its pressure $p_{X}$ to its density $\rho_{X}: w(a) \doteq$ $p_{X}(a) / \rho_{X}(a)$, where $a \doteq 1 /(1+z)$ is the scale factor of the universe (see, e.g., Weinberg 1972). For matter $w=0$, for radiation $w=1 / 3$, and for dark energy this EoS parameter is fundamentally unknown. If one assumes a constant EoS, current observations place limits of $w=-1.06 \pm 0.07$, consistent with the cosmological constant $\Lambda$ (Guy et al. 2010; Conley et al. 2011; Sullivan et al. 2011). However, when the EoS is allowed to evolve with the expansion, observational constraints are much weaker.

A significant challenge in this effort is to devise a physically adequate expression for the evolving dark energy EoS: for it to be useful, $w(a)$ must be sufficiently sophisticated to be able to accommodate the data, and simple enough to provide reliable predictions. As the vast literature on the topic has proved repeatedly, the joint use of SN Ia and other cosmological tools such as baryon acoustic oscillations (BAO; Percival et al. 2010; Beutler et al. 2011; Blake et al. 2011) and the cosmic microwave background (CMB; Komatsu et al. 2011) can help us clarify this picture in the context of a particular cosmological model.

Unfortunately, the signal-to-noise ratio at present is not significant enough to provide satisfactory constraints in more 
than two dark energy parameters (Linder \& Huterer 2005; Sullivan et al. 2008), so for that reason, conclusions do not vary significantly when one considers different parameterizations that are close to each other in a broad sense (smoothness, slow evolution, high-redshift boundedness). Not surprisingly, a representative of this class of parameterizations, the so-called Chevalier-Polarski-Linder (CPL) model (Chevallier \& Polarski 2001; Linder 2003), has become very popular. Here it will be adopted as our reference scenario. This parametrization defines the dark energy EoS as

$w(a)=w_{0}+(1-a) w_{a}$

where $w_{0}$ is the value of the EoS at the present time, and $w_{0}+w_{a}$ is its value at $a=0$, i.e. at redshift $z \rightarrow \infty$. Various attempts to generalize this expression have been made, pointing out the intrinsic difficulty of constraining the evolutionary parameter $w_{a}$ with datasets that are limited in their redshift range (such as SN Ia); or the strong correlation between the two parameters $w_{0}$ and $w_{a}$ (Wang 2008). For a small selection of alternative parameterizations the interested reader is referred to Huterer \& Turner (2001), Barai et al. (2004), Wetterich (2004), Choudhury \& Padmanabhan (2005), Gong \& Zhang (2005), Jassal et al. (2005), Lee (2005), Upadhye et al. (2005), Linder (2006), Lazkoz et al. (2010), and Ma \& Zhang (2011).

The problem of placing observational constraints on $w_{a}$ is perhaps the weakest feature of this parametrization, and the findings in this paper are particularly relevant to this point. Specifically, our results show that a statistically significant number of SN Ia at high redshift $(z>1.0)$ can provide a valuable reduction in the errors on $w_{a}$, i.e. on the asymptotic value of the dark energy EOS. It is well known (Riess \& Livio 2006; Postman et al. 2011) that at $z<1$ SN Ia distance measurements are more sensitive to the static component of the dark energy $w_{0}$; whereas at $1<z<1.5$ the behavior gets reversed and measurements are more sensitive to the dynamic component $w_{a}$. At even higher redshift, $z>1.5$, SN Ia measurements could be more sensitive to peculiarly divergent evolution in the EoS or systematic changes in the SN Ia themselves (if present; Riess \& Livio 2006). Thus, extending SN Ia observations to a higher redshift range than currently available (the maximum redshift in current samples is $z_{\max } \sim 1.39$ ) could be a valuable step toward improving dark energy constraints.

In 2010, the Hubble Space Telescope (HST) embarked on three ambitious multi-cycle treasury (MCT) programs, designed to span three years and produce a lasting archive of deep multi-wavelength imaging. Two of these programs take advantage of the infrared survey capabilities of the new Wide Field Camera 3 (WFC3) to enable the discovery and follow-up of SN Ia out to $z \sim 2.3$ : the Cosmic Assembly Near-infrared Deep Extragalactic Legacy Survey (CANDELS, PIs: Faber and Ferguson; Grogin et al. 2011; Koekemoer et al. 2011), and the Cluster Lensing and Supernova survey with Hubble (CLASH, PI: Postman; Postman et al. 2011). The CANDELS+CLASH SN search program (PI: Riess) comprises the SN search component from both programs. This SN survey is principally aimed at high-redshift $\mathrm{SNe}(z>1.5)$ in order to measure the time dependence of the dark energy equation of state and improve our understanding of SN Ia progenitor systems.

Our goal in this work is to measure the degree of influence that these high- $z$ SN Ia could exert in constraining dark energy parameters. We adopt the CPL parametrization as a good representative of modern dark energy models (Albrecht et al. 2006, 2009), and we use the SuperNova Legacy Survey compilation
(SNLS3; Guy et al. 2010; Conley et al. 2011; Sullivan et al. 2011) as the basis for comparison against future high- $z$ samples.

In Sect. 2 we describe the method we followed to simulate the expected final set from CANDELS+CLASH, and a larger sample representing an extended high- $z$ SN Ia survey with HST. Finally, in Sect. 3 we discuss the results expected for future dark energy constraints and explore some other interesting features that can be achieved only with high- $z$ SN Ia.

\section{Mock data algorithm}

To study the impact of high redshift SN Ia on dark energy constraints, we wanted to extend the SNLS3 samples by adding a synthetic sample of high- $z$ SN Ia that mimics what can be done with HST. To this end we used, in parallel, mock simulations of high-redshift SN Ia and the Fisher matrix formalism. We first realized a Markov chain Monte Carlo (MCMC) analysis, assuming a diagonal covariance matrix and a fixed $\Omega_{\mathrm{m}}$ with our mock data sets (the reasons behind this choice are detailed in the following sections). Then, we performed a Fisher matrix analysis on the same data sets and with the same conditions, thus obtaining a proportionality factor to convert Fisher-derived errors into MCMC-derived ones. As is well known, the Fisher matrix formalism (Tegmark et al. 1997; Huterer \& Turner 2001) only provides a minimum error forecast on the considered quantities because it is based on the ideal hypothesis of Gaussian errors. On the other hand, creating mock data and analyzing them gives more realistic estimations for cosmological constraints (Wolz et al. 2012). We compared errors on the interested parameters derived from a statistical analysis of mock data with those estimated through the Fisher formalism; we were able to find a reliable proportionality between them. This is a useful result, as the Fisher matrix is a more efficient procedure than the MCMC method, and can be easily implemented for any number of SN Ia one wants to consider. Finally, we improved on the initial results with an MCMC analysis performed on the real SNLS3 data set, where we utilized the full covariance matrix, and allowed $\Omega_{\mathrm{m}}$ to be a free parameter, constrained by appropriate priors. Comparing this more rigorous analysis to the results from the previous steps, we found a scaling factor to correct the underestimated cosmological parameter errors into more realistic estimates.

To begin, we first generated our mock SN Ia data, using the following procedure:

1. fit a CPL cosmological model to the real SNLS3 dataset; this will be used as the fiducial cosmological background to produce the mock samples;

2. simulate all the observational quantities and their related errors that enter the SNLS3 data set (for more details, see below), giving us a mock SNLS3 sample;

3. check the accuracy of the simulation procedure by comparing this mock sample with the real one, also performing a cosmological fit with it;

4. use the same algorithm to create the high- $z$ HST mock samples we need, mimicking the expected CANDELS+CLASH yield.

One should expect estimates for the EoS parameters to improve in precision as the sample grows. As discussed in the following section, this procedure for generating mock SN Ia data is designed to avoid systematic biases. Thus, any improvement in precision can be completely attributed to the high-redshift SN Ia. 
Finally, the information used to generate the mock SNLS3 data set (step 2) can be utilized in the Fisher matrix formalism. For a given SN Ia sample, the Fisher matrix approach gives a prediction for the errors on cosmological parameters of interest. We then compare these underestimated Fisher matrix errors to the more realistic errors derived from the mock data analysis. As described in Sect. 2.4, this comparison reveals a tight proportionality that can be used to improve the Fisher matrix results.

\subsection{SNLS3 preliminary considerations}

We first turn our attention to step 1: fitting the SNLS3 data to define our fiducial CPL model. The quantity of interest in the SNLS3 context is the predicted magnitude of the SN Ia $m_{\text {mod }}$ that describes the relative light-curve for any SN Ia given the cosmological model and two other quantities, the stretch (a measure of the shape of the SN Ia light-curve) and the color. It reads

$m_{\text {mod }}=5 \log _{10}\left[\mathrm{~d}_{L}\left(z, \Omega_{\mathrm{m}} ; \theta\right)\right]-\alpha(s-1)+\beta C+\mathcal{M}$,

where $\alpha$ and $\beta$ are the parameters that characterize the stretchluminosity and color-luminosity relationships, reflecting the well-known broader-brighter and bluer-brighter relationships, respectively (Conley et al. 2011). It depends on $\mathrm{d}_{L}\left(z, \Omega_{\mathrm{m}} ; \theta\right)$, the Hubble free luminosity distance

$\mathrm{d}_{L}\left(z, \Omega_{\mathrm{m}} ; \theta\right)=(1+z) \int_{0}^{z} \frac{\mathrm{d} z^{\prime}}{E\left(z^{\prime}, \Omega_{\mathrm{m}} ; \theta\right)}$

with the Hubble function $E(z)=H(z) / H_{0}$ depending on the matter and dark energy components and $\theta$ being the EoS parameters vector $\left(\theta=\left(w_{0}, w_{a}\right)\right.$ for the CPL cosmological model $) ; \mathcal{M}$ is a nuisance parameter combining the Hubble constant $H_{0}$ and the absolute magnitude of a fiducial SN Ia. This notation is chosen to clarify the roles of the various cosmological parameters appearing in the formulae. The fractional matter density parameter $\Omega_{\mathrm{m}}$ is separated as we fix it to an a priori value $\Omega_{\mathrm{m}}=0.259$, derived from a quiessence model analysis of WMAP7.1 CMB data ${ }^{1}$.

By assuming a fixed $\Omega_{\mathrm{m}}$ rather than leaving it as a free parameter, we can vastly simplify the generation of our mock data sets. This is important because, as we will discuss in the next section, we have a large number of parameters contributing to the total error budget that we have to simulate. By fixing $\Omega_{\mathrm{m}}$ we can focus our analysis on observing the effects in $w_{0}$ and $w_{a}$. Unfortunately, this assumption necessarily leads to an underestimation of the errors for these dark energy parameters. In Sect. 2.4 we describe the procedure that we have implemented to address this shortcoming, which recovers a more realistic estimation of the $w_{0}$ and $w_{a}$ uncertainties.

Equations (2) and (3) can be used after defining a cosmological model to be tested. As we will fit our SN Ia data with the CPL model, the Hubble function $E(z)=H(z) / H_{0}$ will depend on matter and dark energy components through the expressions

$E(z)=\left[\Omega_{\mathrm{m}}(1+z)^{3}+\left(1-\Omega_{\mathrm{m}}\right) X(z, \theta)\right]^{1 / 2}$

and

$X(z, \theta)=\exp \left[3 \int_{0}^{z} \frac{\mathrm{d} z^{\prime}}{1+z^{\prime}}\left(1+w\left(z^{\prime}, \theta\right)\right)\right]$.

\footnotetext{
1 http://lambda.gsfc.nasa.gov/product/map/current/ params/wcdm_sz_lens_wmap7.cfm
}

Here we have assumed spatial flatness. Using the CPL parametrization we get

$X(z)=(1+z)^{3\left(1+w_{0}+w_{a}\right)} \exp \left[-\frac{3 w_{a} z}{1+z}\right]$.

There is an important difference in the SNLS3 handling of the parameters $\alpha$ and $\beta$ which enter in Eq. (2) with respect to other SN Ia samples in the literature, such as the Union2 data set (Amanullah et al. 2010). In the SNLS3 approach these parameters are left free during all stages of their error estimation, both for statistical and systematic uncertainties; in other cases (Amanullah et al. 2010), the authors find best-fit values for $\alpha$ and $\beta$ in the preliminary stages, when building the SN Ia sample, and then hold $\alpha$ and $\beta$ at those fixed values for uncertainty calculations in the cosmological analysis. In Conley et al. (2011) the authors demonstrate that this approach can introduce a bias in the determination of the cosmological parameters.

Furthermore, the SNLS3 team ${ }^{2}$ advocates the use of all the components (both statistical and systematic errors) of the covariance matrix. Most importantly for our purposes, they provide data files with the full multidimensional covariance matrix for all the physical quantities involved in their analysis, allowing us to reconstruct the contributions of first-order covariance terms to the uncertainties (see Sect. 2.2). As the main purpose of this work is to detect the relative decrease of errors (if any) on cosmological parameters by adding simulated high-redshift SN Ia from HST, we made two choices: (1) we have left $\alpha$ and $\beta$ free as prescribed in Conley et al. (2011); and (2) we use only diagonal components of the statistical and systematic covariance matrix, because of the intrinsic difficulty in simulating out-ofdiagonal terms of the covariance matrix (see Sect. 2.2). In this way, the expected underestimation of real errors on cosmological parameters (Sullivan et al. 2011) due to the sole use of statistical observational errors is highly softened. Moreover, this does not affect the primary goal of this work, which is to quantify the relative decrease of errors on cosmologically interesting dark energy parameters.

\subsection{Generating mock data and errors}

To generate our mock SNLS3 catalog of $m_{\bmod }\left(z_{i}\right)$ values, we use $m_{\text {mod }}\left(z_{i}\right)=m_{\text {mod,CPL }}\left(z_{i}\right)+\Delta m_{\text {mod,CPL }}\left(z_{i}\right)$,

where $z_{i}$ with $i=1, \ldots, \mathcal{N}_{\text {data }}$ and $\mathcal{N}_{\text {data }}=472$ for SNLS3, are randomly drawn redshift values selected to match the true redshift distribution in the real SN Ia samples; $m_{\text {mod,CPL }}$ is the value of the magnitude at a given redshift calculated from the best fit of the SNLS3 sample with a CPL model. It is important to underline here that in order to calculate the fiducial $m_{\text {mod,CPL }}$ value we also have to simulate the stretch and color quantities. The $\alpha$ and $\beta$ parameters are derived from the best-fit analysis; and the nuisance parameter $\mathcal{M}$ can be calculated following Conley et al. (2011).

The offset $\Delta m_{\text {mod,CPL }}$ is a random noise component added to $m_{\text {mod,CPL }}$ in order to give the mock dataset a realistic dispersion (i.e. similar to the real datasets). This random noise is sampled, for any redshift, from a Gaussian distribution with zero mean and standard deviation $\sigma_{m_{B}}^{\text {eff }}$, defined below as the total magnitude-only error.

While it is relatively easy to produce mock values for the SN Ia magnitudes, more attention must be paid to the reproduction of errors on $m_{\bmod }\left(z_{i}\right)$. Here we emphasize again that we are

2 http://www.cfht.hawaii.edu/SNLS/ 

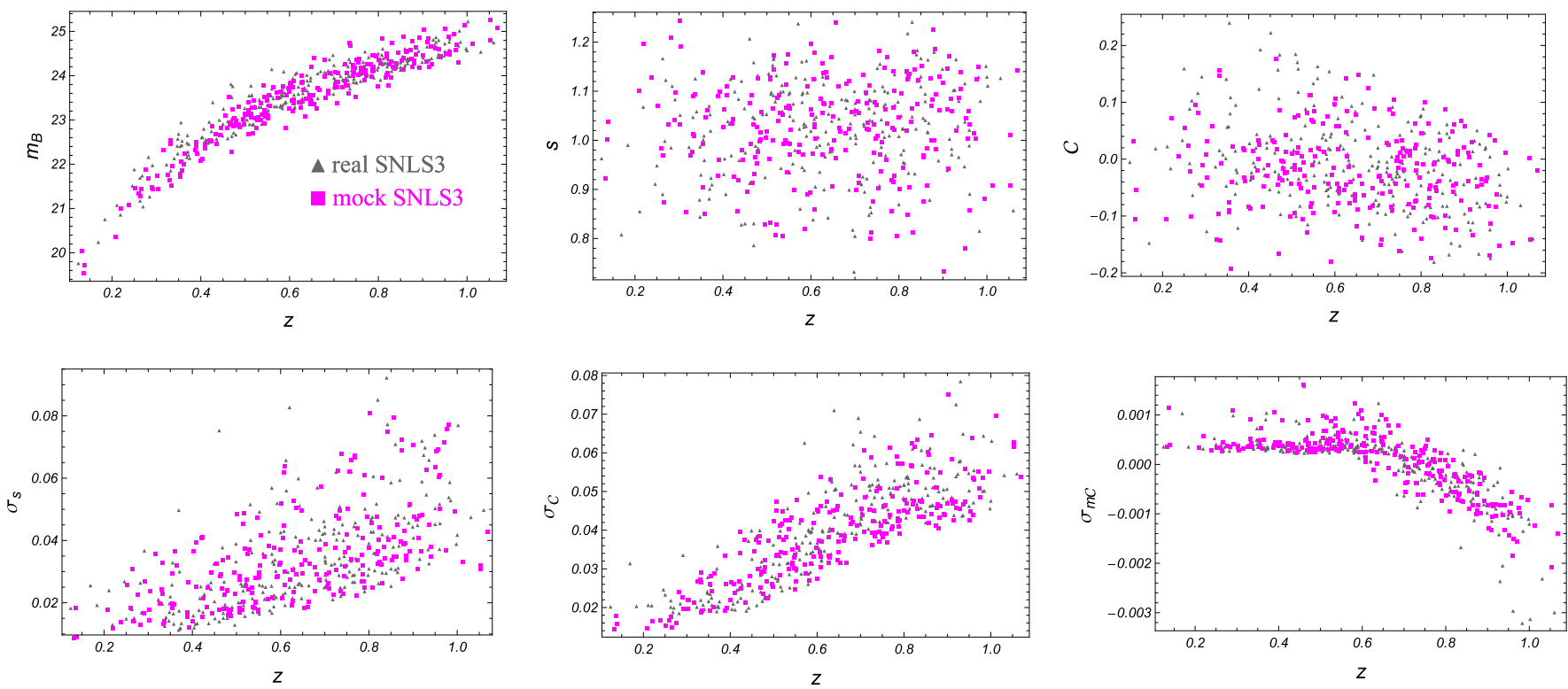

Fig. 1. Comparing real and mock SNLS sub-sample from the SNLS3 data set: gray triangles are for real SNLS3 data, magenta squares for mock SNLS3 data. Top. From left to right: magnitude, stretch and color vs. redshift. Bottom. From left to right: diagonal stretch error, color error, and magnitude-color covariance vs. redshift.

using only the diagonal part of the total covariance matrix. The statistical errors on the magnitude $m_{\text {mod }}$ are given by the relation (Conley et al. 2011)

$$
\begin{aligned}
\sigma_{\text {stat }}^{2}= & \sigma_{m_{B}}^{\mathrm{eff}, 2}+\alpha^{2} \sigma_{\mathrm{s}}^{2}+\beta^{2} \sigma_{\mathrm{C}}^{2}+2 \alpha \sigma_{\mathrm{ms}}-2 \beta \sigma_{\mathrm{mC}} \\
& -2 \alpha \beta \sigma_{\mathrm{sC}}+\sigma_{z}^{2},
\end{aligned}
$$

with

$\sigma_{m_{B}}^{\mathrm{eff}, 2}=\sigma_{\mathrm{int}}^{2}+\sigma_{\mathrm{pec}}^{2}+\sigma_{m_{B}}^{2}$.

The different quantities contributing to $\sigma_{\text {stat }}$ are described as follows: $\sigma_{m_{B}}$ is the error on the observed magnitude including lensing and host galaxy effects; $\sigma_{\mathrm{s}}$ and $\sigma_{\mathrm{C}}$ are the errors on the stretch and color parameters; $\sigma_{\mathrm{ms}}, \sigma_{\mathrm{mC}}$, and $\sigma_{\mathrm{sC}}$ are the covariance terms between magnitude, stretch, and color; $\sigma_{\text {int }}$ is the intrinsic scatter of SN Ia and it is different for every sub-sample; $\sigma_{\text {pec }}$ is the peculiar velocity error and $\sigma_{z}$ is the error on redshift converted into magnitude uncertainty (see Conley et al. 2011, for more details on their calculation).

This is only a part of the total covariance matrix; also taking into account the systematic terms, this matrix would be

$$
\begin{aligned}
\mathbf{C}= & \sigma_{\text {stat }}^{2}+\sigma_{\text {syst }, m_{B}}^{2}+\alpha^{2} \sigma_{\text {syst, } \mathrm{s}}^{2}+\beta^{2} \sigma_{\text {syst,C }}^{2} \\
& +2 \alpha \sigma_{\text {syst,ms }}^{2}-2 \beta \sigma_{\text {syst,mC }}^{2}-2 \alpha \beta \sigma_{\text {syst }, \mathrm{sC}}^{2}
\end{aligned}
$$

where the bold font is used for matrices, the suffixes refer to the statistical or systematic nature of the errors, and other symbols have the same meaning as in Eq. (8).

The difficulty in building mock error samples lies in the dependence of the statistical errors on the free parameters $\alpha$ and $\beta$; for this reason we cannot attempt a global phenomenological fit of them. To fully describe the total statistical and systematic errors we have to generate mock sub-samples survey by survey (as they are given in Table 3 of Conley et al. 2011) for all the terms on the right sides of Eqs. (8)-(10). For most of the SNLS3 subsamples, we find a good fit for the error terms by assuming a power law $\propto(1+z)^{n}$, generally obtaining $n \sim 1$ or $\sim 2$. However, in those cases where no clear pattern was detectable we have opted for a random distribution (independent of redshift) which closely mimics the real data. For the $\sigma_{m_{B}}^{2}$ term, which contains some redshift dependent elements, we have performed fits following the relation given by (Kim \& Linder 2011), $\sigma_{\mathrm{s}}^{2}\left(\frac{1+z}{1+z_{\max }}\right)^{2}$, where $z_{\max }$ is the maximum observable redshift for each survey.

\subsection{Testing the goodness of the mock building algorithm}

Despite the high number of parameters entering the definition of the total covariance matrix, we can see in Figs. 1 and 2 that our simple empirical approach does yield mock quantities and errors that match the true distributions quite reliably (for illustrative purposes only, in Fig. 2 we have fixed $\alpha$ and $\beta$ to the best-fit values from the cosmological fit of real SNLS3).

To firmly validate the quality of our mock procedure, we also compare the outcomes of cosmological fits to real and mock SNLS3 data sets. These fits have been obtained by minimizing the quantity

$\chi^{2}(\theta)=\sum_{j=1}^{\mathcal{N}_{\mathrm{SN}}} \frac{\left(m_{B}\left(z_{j}\right)-m_{\mathrm{mod}}\left(z_{j}, \Omega_{\mathrm{m}} ; \alpha, \beta, \theta\right)\right)^{2}}{C_{j}}$.

We marginalize over the nuisance parameter $\mathcal{M}$ following the prescription given in Conley et al. (2011). Recent empirical evidence suggests two different values of $\mathcal{M}$ depending on the value of the host stellar mass, so we define two sub-samples, one with $\log M_{\text {host }}<10$ and the other with $\log M_{\text {host }}>10$. The expression for this $\chi^{2}$ marginalized over $\mathcal{M}$ is given in Appendix $\mathrm{C}$ of Conley et al. (2011). To minimize the $\chi^{2}$ we use the MCMC method and test for convergence with the method described by Dunkley et al. (2005).

The comparison between the real and mock SNLS3 in Fig. 3 shows a slight shift in both cosmological parameters, although the errors are almost the same. This is the price to be paid (in addition to the usual problems of mock procedures) for not fitting the total errors directly: as we have discussed in the previous section, we have to fit each term contributing to the error and 
V. Salzano et al.: Dark energy constraints with high-redshift CANDELS and CLASH supernovae
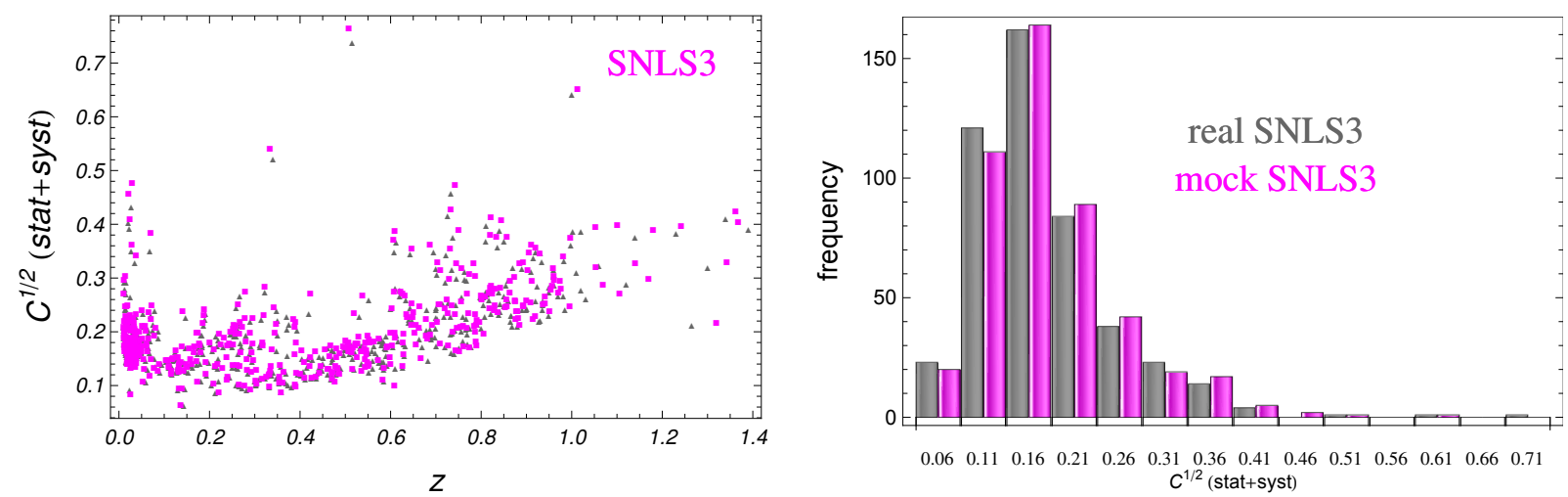

Fig. 2. Left: total statistical plus systematic diagonal errors on SN Ia magnitude vs. redshift: light-gray triangles are for real SNLS3 data, magenta squares for mock SNLS3 data. Right: comparison of histograms for the same errors from the real and mock SNLS3 datasets (gray and magenta bars, respectively).

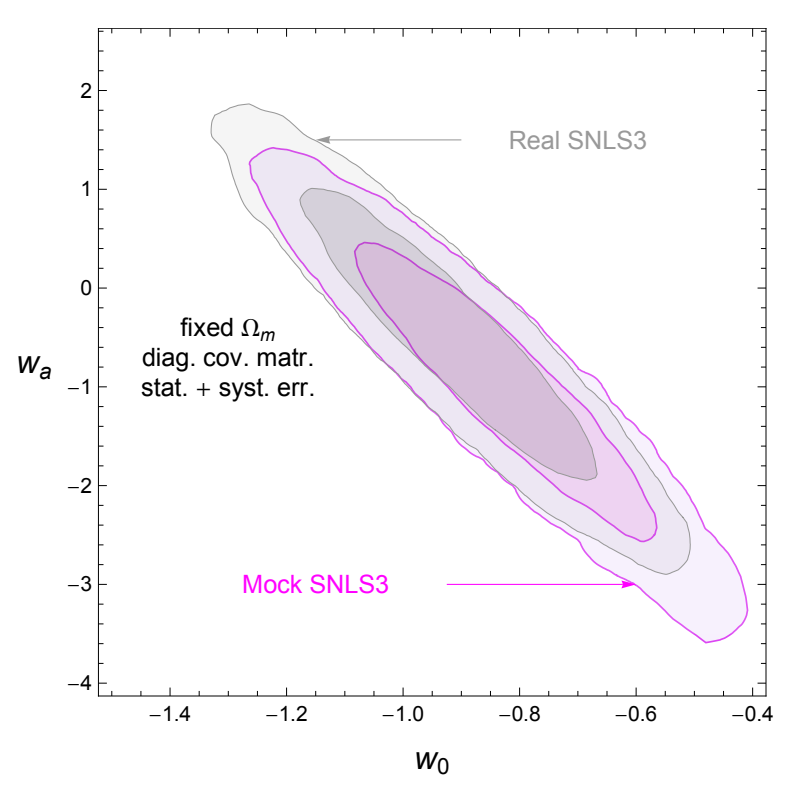

Fig. 3. Fitting the CPL model with SN Ia data: $1 \sigma$ and $2 \sigma$ contours in the $w_{0}-w_{a}$ parameter space. Gray contours are from the real SNLS3 data set, magenta contours from the mock SNLS3 data set. Contours reflect statistical plus systematic errors.

each of them contributes with its dispersion to the final results. Moreover, we have two more parameters, $\alpha$ and $\beta$ in the fit, which can influence the final results by interacting with the stretch and the color parameters and with the error expression. But we underline that an absolute comparison of results is beyond the scope of this work; what is important is to verify that the cosmological parameter uncertainties are of the same order (if not almost perfectly coincident) and that the contours are equivalent regardless of the small shift. A way to quantify this strong agreement is the FoM, defined as the inverse of the area of the contours: for the mock SNLS3 data set we get a FoM $\lesssim 4 \%$ smaller than its real counterpart (Col. 4 in Table 2). This result validates our approach and is important for three main reasons. First, it means that our mock observational quantities and errors are not losing any information that may be included in the real data; second, it justifies the generation of the synthetic CANDELS+CLASH sub-sample of SN Ia by the same procedure; and third, our data can be appropriately used as input in the Fisher formalism that we will discuss in the next section.
Table 1. Mock samples.

\begin{tabular}{lcc}
\hline \hline Redshift range & \multicolumn{2}{c}{ Number of SN Ia ${ }^{a}$} \\
\hline & $\begin{array}{c}\text { CANDELS+CLASH } \\
\text { (HST/3 yrs) }\end{array}$ & $\begin{array}{c}\text { HST+WFC3 6 yr } \\
\text { (HST/6 yrs) }\end{array}$ \\
\hline $1.0<z<1.5$ & 8 & 16 \\
$1.5<z<2.0$ & 5 & 10 \\
$2.0<z<2.5$ & 1 & 2 \\
\hline Total: & 14 & 28 \\
\hline
\end{tabular}

Notes. ${ }^{(a)}$ Number of cosmologically useful SN Ia expected to be discovered by the three-year CANDELS+CLASH program and an imagined extension into a six-year program with HST+WFC3, including CANDELS+CLASH and another similar three-year survey.

\subsection{Mock CANDELS+CLASH}

In Table 1 we define two plausible distributions of high-redshift SN Ia events that could be observed with HST+WFC3. The $\mathrm{HST} / 3$ yrs mock sample is designed to mimic the expected yield from the three-year SN Ia survey of CANDELS+CLASH, with $14 \mathrm{SN}$ Ia at high-redshift (i.e., at $z>1$ ) out to $z=2.5$ that are useful for cosmology (i.e. with light curves). The HST/6 yrs mock sample imagines a future survey with WFC3 that extends the HST SN Ia detections for another three years, doubling the sample to $28 \mathrm{SN}$ Ia out to $z=2.5$.

For each of these samples we must extrapolate from existing data to predict the distance modulus uncertainties in the new redshift regime. We extrapolate from the SNLS3 sub-sample containing 17 SN Ia observed with HST using the Advanced Camera for Surveys (ACS). In this way, statistical and systematic errors in the mock sample should represent a realistic yet conservative scenario. This final set of two mock samples now allows us to efficiently study the impact that a high- $z$ SN Ia sample from HST could have in constraining the dark energy EoS.

These HST/ 3 yrs and HST/6 yrs mock data sets are the two main tools we use for our analysis. We will also consider larger samples (up to 100 high-redshift SN Ia) of the same kind in order to discern if there is a simple relationship between the cosmological parameter errors that come from mock SN Ia samples and those derived through the Fisher matrix formalism. As is well known, the inputs for a Fisher procedure are a fiducial cosmological model, the redshift distribution of the sample, and the error prescriptions for the survey(s) being considered. For the fiducial cosmological model we use the best-fit CPL model from our fit to the real SNLS3 data. The redshift distribution 
Table 2. SNLS3: errors for the CPL cosmological model using the assumptions and the datasets described in the text.

\begin{tabular}{lccc}
\hline \hline & \multicolumn{3}{c}{ Prior $\Omega_{\mathrm{m}}+$ full cov. } \\
\hline SNLS3 & $\sigma_{w_{0}}$ & $\sigma_{w_{a}}$ & FoM \\
real & 0.213 & 1.647 & 4.92 \\
mock & 0.224 & 1.722 & 4.49 \\
real+HST/3 yrs & 0.198 & 1.430 & 5.91 \\
real+HST $/ 6$ yrs & 0.187 & 1.305 & 6.82 \\
real $+N_{z}>1=56$ & 0.177 & 1.182 & 7.85 \\
real $+N_{z}>1=126$ & 0.164 & 1.010 & 9.51 \\
real + HST $/ 6$ yrs + JWST & 0.171 & 1.123 & 8.93 \\
\hline
\end{tabular}

Notes. Results on each parameter follow from marginalization over the other parameters of the model. Column 1: dataset used. Column 2: $1 \sigma$ confidence level for $w_{0}$. Column 3: $1 \sigma$ confidence level for $w_{a}$. Column 4: figure of merit for any dataset.

corresponds to the redshift values from the real SNLS3, with extensions to higher redshift as given in Table 1 . The errors are defined by the real SNLS3 data and the mock HST/3 yrs and HST/6 yrs samples.

With this input, the Fisher matrix procedure returns an estimate of the errors on the cosmological parameters of interest (the Fisher errors on $w_{0}$ and $w_{a}$ ). We then compare these to the errors derived from fitting a CPL model to the mock data (the mock errors). We find that the ratio of Fisher errors to mock errors is constant for all sample sizes (as long as the errors on cosmological parameters are very likely Gaussian and with very small asymmetries). If we define this constant $p_{i} \doteq \frac{\sigma_{i}^{\text {mock }}}{\sigma_{i}^{\text {Fisher }}}$ (with $i=w_{0}$, $w_{a}$ ) we have $\left\langle p_{w_{0}}\right\rangle=1.59$ and $\left\langle\Delta p_{w_{0}}\right\rangle=0.03 ;\left\langle p_{w_{a}}\right\rangle=1.15$ and $\left\langle\Delta p_{w_{a}}\right\rangle=0.02$. This result allows us to scale up all the Fisher errors (which are understood to be lower limits on the true uncertainty) into a more realistic estimation of the $w_{0}$ and $w_{a}$ uncertainties, but not yet completely realistic.

As noted in Sects. 2.1 and 2.2, we expect that these uncertainties are still significantly underestimated because of two key simplifications we have used: assuming a fixed $\Omega_{\mathrm{m}}$, and leaving out the off-diagonal elements of the covariance matrix. To see how these choices influence our error estimates, we have performed an MCMC analysis of the real SNLS3 data set with the total covariance matrix given by Conley et al. (2011). Here we have not fixed $\Omega_{\mathrm{m}}$, but instead apply a Gaussian prior on the matter content $\Omega_{\mathrm{m}}=0.26 \pm 0.02$ (Wang 2011). This prior includes information from external cosmological data sets other than SN Ia. We then repeat the analysis on the real SNLS3 data, but now using our simplification of a fixed $\Omega_{\mathrm{m}}$ and only diagonal covariance terms. Comparing the $w_{0}$ and $w_{a}$ errors returned from these two approaches gives us an estimate of the systematic bias in $w_{0}$ and $w_{a}$ that may be introduced by our simplifying assumptions. From this comparison we derive a proportionality factor, which we then apply as a multiplicative correction for $\sigma_{w_{0}}$ and $\sigma_{w_{a}}$. In this way we are left with estimations of the dark energy EoS uncertainties that are much more realistic and reliable.

\section{Results and conclusions}

Let us outline our main findings, keeping in mind that our fits have been carried out under the assumption of a CPL model. Figure 4 and Table 2 give a quantitative summary showing that, as expected, the high-redshift SN Ia reduce the errors on the EoS parameters in all cases. This effect is small for $w_{0}$, which is already quite narrowly constrained by present data: we see a reduction in $\sigma_{w_{0}} \approx 7-12 \%$. However, the improvement is more pronounced in the case of the dark energy evolution parameter $w_{a}$ with a reduction in $\sigma_{w_{a}} \approx 13-21 \%$. The narrower constraints on $w_{0}$ and $w_{a}$ induce in turn a significant improvement in the FoM, increasing by $20-39 \%$.

\subsection{Future prospects}

We can now look beyond the immediate horizon of the current CANDELS+CLASH HST surveys, and examine how much the precision in the measurement of $w_{0}$ and $w_{a}$ can plausibly be improved by adding more high- $z$ SN Ia. We can also ask a broader question. How many high- $z$ SN Ia from space-based surveys would be needed to provide a noticeable improvement in our measurement of these cosmological parameters?

Final results (Fig. 5) show that adding high-redshift SN Ia, with the CANDELS+CLASH redshift distribution we have considered in this work, can produce a substantial decrease in the statistical and systematic errors on $w_{0}$ and $w_{a}$ up to a maximum of $\mathcal{N}_{z>1} \sim 56$. This approximately corresponds to a ten-year campaign in the mold of CANDELS+CLASH (almost certainly exceeding the remaining lifetime of HST). This imagined sample of $56 \mathrm{SN}$ Ia would decrease the uncertainty in $w_{0}$ by $\approx 5 \%$ improvement relative to the HST/6 yrs benchmark simulation (equivalently, a $\approx 17 \%$ improvement compared to the present status). For $w_{a}$ the projected improvement is $\approx 7 \%$ relative to the HST $/ 6$ yrs expectation (or $\approx 28 \%$ compared to the present status). Beyond this point the rate of decrease of the errors starts to slow down. To obtain another reduction in errors of the same magnitude, one would need to more than double the high- $z$ SN Ia sample $\left(\mathcal{N}_{z>1}>120\right)$. Further increases in the sample size produce no appreciable improvement.

With $\sim 56 \mathrm{SN}$ Ia the projected total error (statistical plus systematic) on $w_{0}$ and $w_{a}$ at the end of this supposed tenyear HST program would be approximately $\sigma_{w_{0}}<0.18$ and $\sigma_{w_{a}}<1.2$. These projected uncertainties from mock SN Ia alone happen to be very comparable to the total uncertainty that can presently be achieved by combining SN constraints with other cosmological probes. For example, (Sullivan et al. 2011) use the SNLS3 SN compilation combined with measurements of the Hubble constant, the cosmic microwave background, and baryon acoustic oscillations. They find very similar uncertainties, $\sigma_{w_{0}} \sim 0.19$ and $\sigma_{w_{a}} \sim 1.1$.

\subsection{SN la evolution}

In the next decade, ground-based SN Ia samples will continue to grow, with wide-field surveys such as Pan-STARRS ${ }^{3}, \mathrm{DES}^{4}$, and $\mathrm{LSST}^{5}$ providing several thousand SN Ia at $z<1.5$. In this environment the real improvement in cosmological constraints from high- $z$ SN Ia samples like CANDELS+CLASH may come from the ability to test for as-yet-unseen systematic biases in the SN Ia sample.

At low redshift $(z<1.5)$ the average SN Ia that we observe will have begun its life as a progenitor star with a main sequence (MS) mass $M_{\mathrm{MS}} \sim 1 M_{\odot}$. However, at high redshift,

\footnotetext{
3 Pan-STARRS: the Panoramic Survey Telescope and Rapid Response System; http://www.ps1sc.org/index.shtml

4 DES: the Dark Energy Survey; http://www. darkenergysurvey. org/

5 LSST: the Large Synoptic Survey Telescope; http://www.lsst. org/lsst/
} 
V. Salzano et al.: Dark energy constraints with high-redshift CANDELS and CLASH supernovae
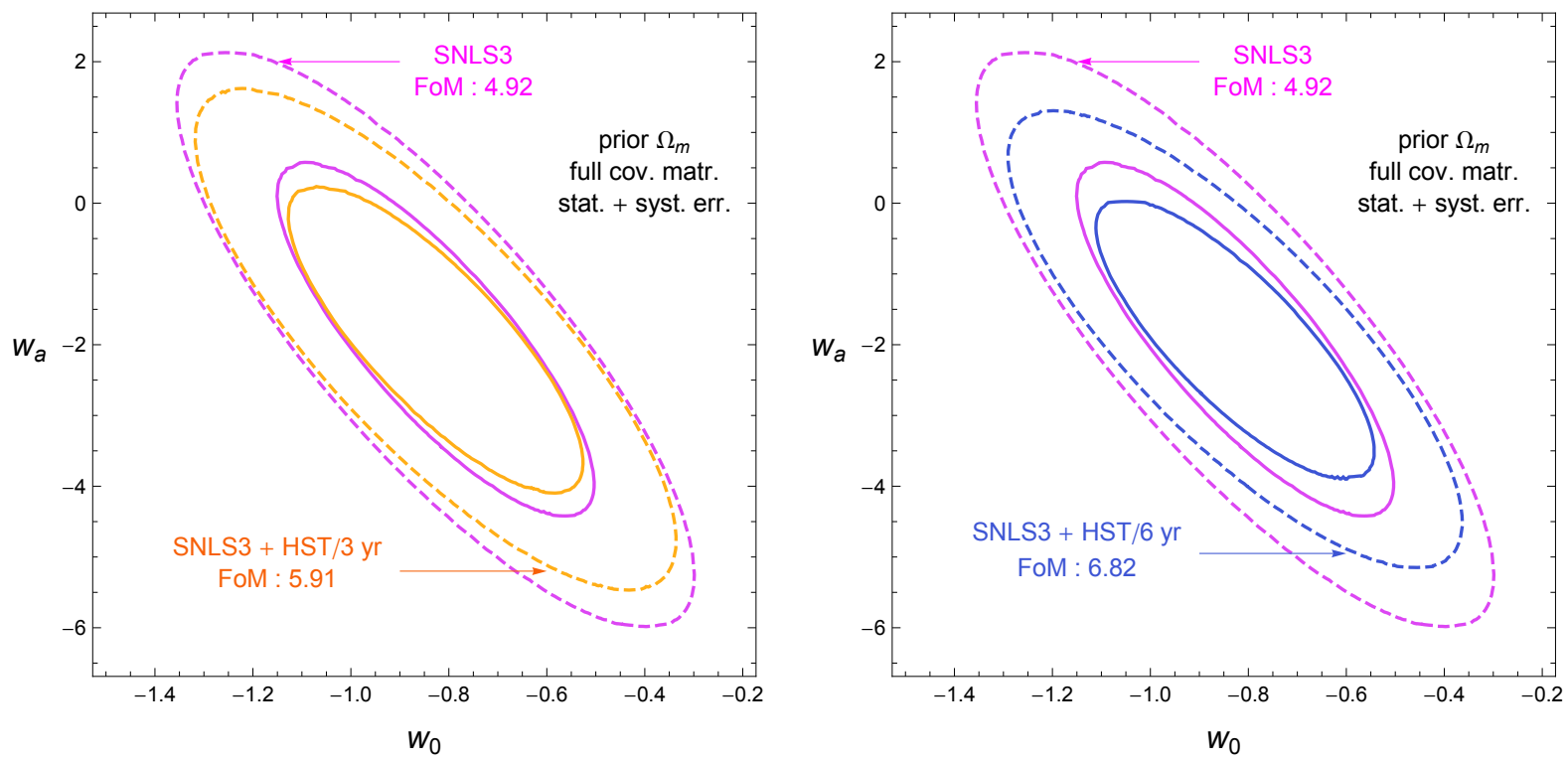

Fig. 4. CPL model with SN Ia data: $1 \sigma$ and $2 \sigma$ contours in the $w_{0}-w_{a}$ parameter space. Left panel: magenta contours come from the SNLS3 data set, red contours from the SNLS3+HST/3 yrs mock data set. Right panel: magenta contours come from the SNLS3 data set, blue contours from the SNLS3+HST/6 yrs mock data set.
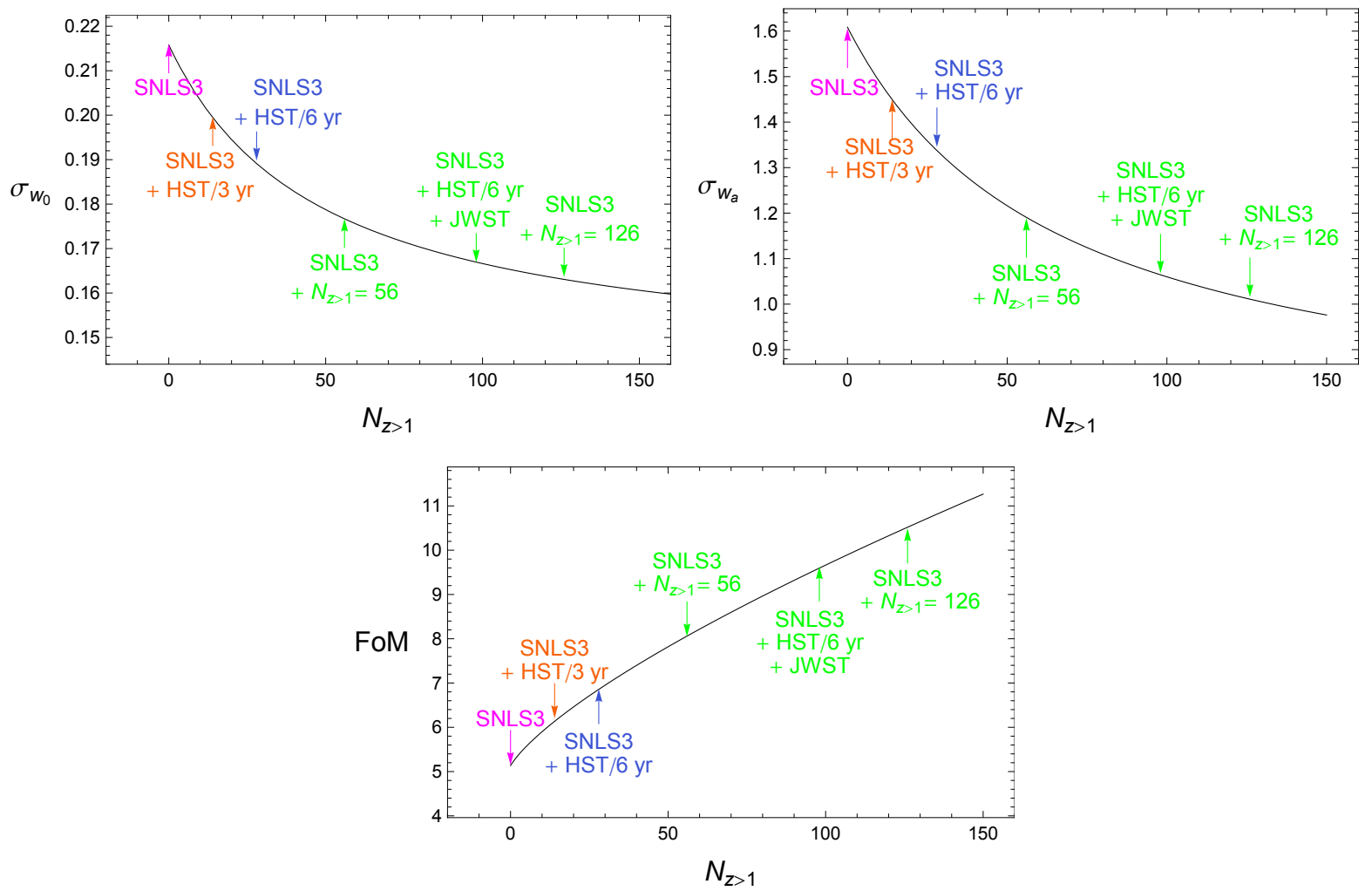

Fig. 5. Future perspective analysis of SN Ia data. $1 \sigma$ errors in the $w_{0}-w_{a}$ parameter vs. number of high-redshift SN Ia : magenta - present SNLS3 data set; red - mock SNLS3+HST/3 yrs-like data set; blue - mock SNLS3+HST/6 yrs-like data set; green - forecasts for various number of high redshift SN Ia.

when the universe was young, these low-mass stars are still on the main sequence, so they cannot contribute to the observed high- $z$ SN Ia population. Thus the mean initial progenitor mass for the observable SN Ia population increases with redshift. Following Riess \& Livio (2006), we adopt the progenitor mass - luminosity relationship of (Dominguez et al. 2001, hereafter DHS2001) as a plausible example of how this demographic shift could systematically bias the observed SN Ia distances and therefore cosmological parameters. The DHS2001 model suggests that more massive SN Ia will be slightly fainter, which we might detect by seeing the average SN Ia peak magnitude shift systematically fainter than the baseline cosmological model as redshift increases. Using our mock high- $z$ SN Ia samples, we can examine how such models for SN Ia evolution could be confronted by current and future surveys.

In addition to the MS lifetime, each SN Ia progenitor system must undergo some period of post-MS mass transfer. We consider two simplistic assumptions for this post-MS timescale: in 

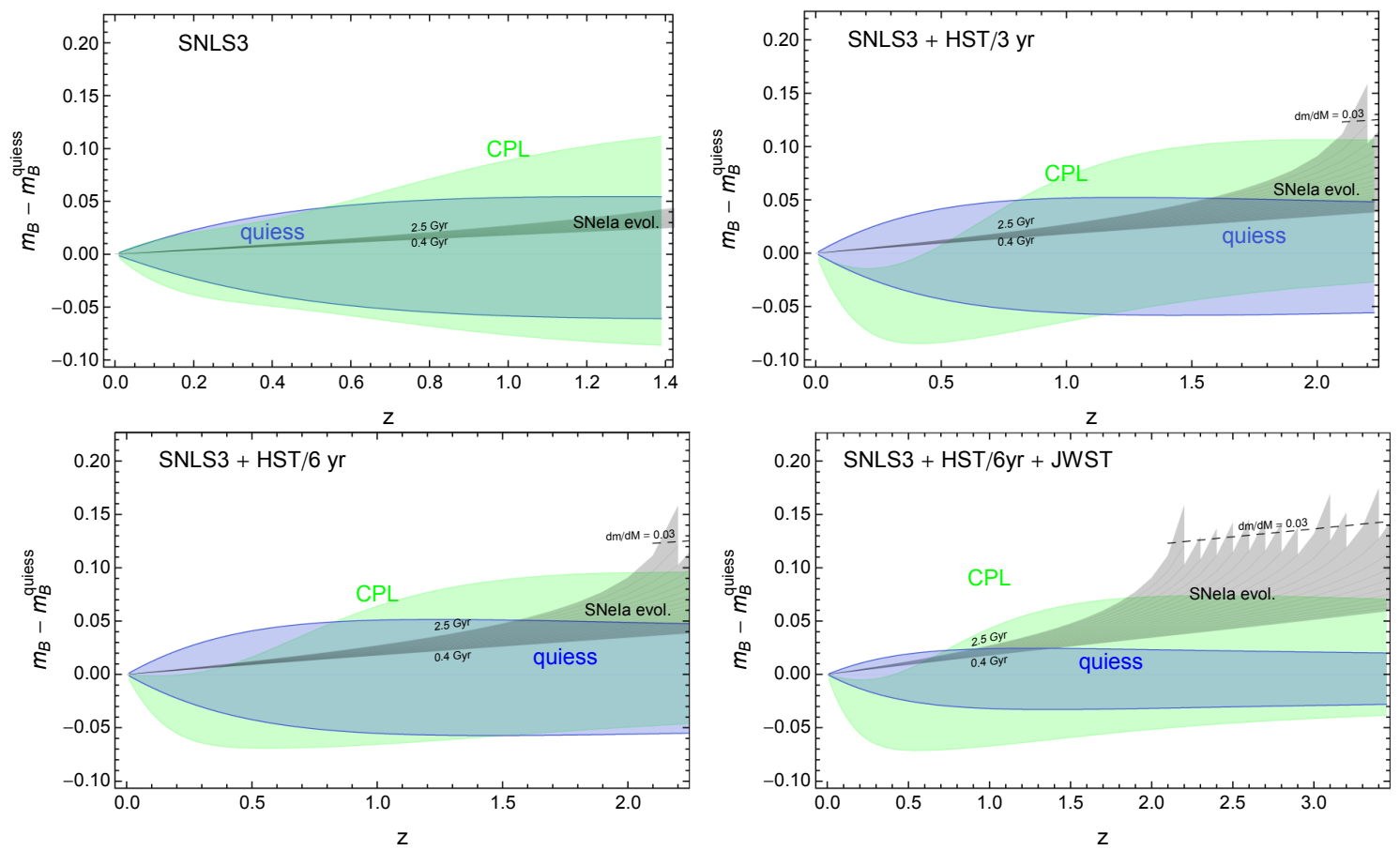

Fig. 6. Future tests for SN Ia evolution. Blue contours show the $1 \sigma$ confidence levels derived from errors on the equation of state parameter $\left(w_{0}\right)$ of quiessence models. Light green regions are the $1 \sigma$ confidence levels derived from errors on $w_{0}$ and $w_{a}$ of CPL models. Gray regions denote a plausible range for SN Ia evolution effects, following Dominguez et al. (2001) and Riess \& Livio (2006) and assuming $\mathrm{d} m_{B} / \mathrm{d} M_{\mathrm{MS}} \sim 0.03$.

case 1 we assume that all SN Ia progenitors require $\tau=2.5 \mathrm{Gyr}$ after leaving the main sequence to reach the point of explosion; for case 2 we use $\tau=0.4$ Gyr. In reality there is a distribution of delay times, but these two extrema can serve to bracket a broad range of plausible progenitor models. To define the mean initial mass of a SN Ia progenitor as a function of redshift, we adopt a Salpeter initial mass function (IMF), and truncate the low-mass end by removing any stars with $M_{\mathrm{MS}}<0.6 M_{\odot}$ (these would never accrete enough mass to reach the Chandrasekhar mass limit and explode) and also removing any for which the combined MS and post-MS timescales exceed the age of the universe since $z=11$. This is a slightly conservative prescription, as it implicitly assumes that all SN Ia progenitor stars formed at $z=11$, although in fact many would have formed later and would therefore also still be on the MS or accreting towards the Chandrasekhar limit. We also truncate the high mass end of the IMF, removing stars with $M_{\mathrm{MS}}>8.0 M_{\odot}$ (these would result in core collapse supernovae instead of SN Ia).

Now with the doubly truncated IMF at each redshift $z$, we can compute the mean SN Ia progenitor mass at each redshift. The DHS2001 model predicts that an increase in the initial progenitor mass of $1 M_{\odot}$ would reduce the peak $B$ or $V$ band brightness by $\sim 0.03$ mag without a corresponding change in the color or light curve shape $\mathrm{d} m_{B} / \mathrm{d} M_{\mathrm{MS}} \sim 0.03$. This converts the expected change in the mean initial mass into a systematic shift of the average peak magnitude for the high- $z$ SN Ia population.

To see if this effect would be distinguishable from dark energy evolution, we have analyzed our data sets with a quiessence cosmological model, which is cosmologically-time independent (constant equation of state). If we then calculate the residuals between SN Ia magnitudes and this reference cosmological model and, if there is any evolutionary effect, it should be detectable as an excess in magnitude. Nevertheless, we have to consider that we would have at least two possible evolutionary phenomena of different natures: one would be related to the cosmological evolution (it would be, for example, the difference between a quiessence and a CPL model); and the other would be linked to the SN Ia evolution which we would like to detect. In Fig. 6 we show blue contours representing the $1 \sigma$ confidence levels derived from errors on the equation of state parameter ( $w_{0}$-quiessence). Green contours show the difference between the CPL and quiessence models, representing the range of SN Ia magnitudes that is consistent with our current ignorance of dark energy evolution. Finally, the grey regions show the posited SN Ia evolutionary effect; if the grey regions overlap the green CPL contours, then the two effects would be indistinguishable.

With the present data, SN Ia evolution is clearly indistinguishable from a quiessence model if $\mathrm{d} m_{B} / \mathrm{d} M_{\mathrm{MS}} \sim 0.03$ and, given the uncertainty regarding cosmological evolution, it is not possible to disentangle the two phenomena. The situation will not change when moving to a HST 3 yrs -like survey; in this case, even if the uncertainty from cosmological evolution is smaller, it is again too large to disentangle the two phenomena, and only SN Ia with $z>2.1$ could be helpful in this direction, even if they would not be numerous enough to be considered statistically significant. Adding more observations (HST/6 yrslike survey) improves the constraint on CPL parameters, thus lowering again the minimum redshift useful for the detection of SN Ia evolution $(z \gtrsim 2)$.

In the bottom panel of Fig. 6 we also plot a very likely future scenario. We can consider the impact of adding to the HST legacy with a high- $z$ SN Ia survey using the James Webb Space Telescope $^{6}$ (JWST); using the CANDELS+CLASH survey as a model, we estimate that a three-year survey with JWST could yield $\sim 36 \mathrm{SN}$ Ia at $1.5<z<3.5$. Adding this to the HST/6 yrs sample further improves constraints on the CPL model (see last line in Table 2). The result is that $\mathrm{SN}$ Ia down to $z \approx 1.7$ could become useful probes for SN Ia evolution models, and even a model with $\mathrm{d} m_{B} / \mathrm{d} M_{\mathrm{MS}} \sim 0.03$ could be, in principle,

6 http://www.jwst.nasa.gov/ 
detectable. In closing, we note that these are probably conservative estimates: low- $z$ SN Ia surveys (e.g. PS1, DES, LSST) should also improve the constraints on CPL models in the intervening decade, thus making the HST and JWST high- $z$ SN Ia even more powerful as tests of SN Ia evolution models.

Acknowledgements. We thank the anonymous referee for comments that greatly improved the quality and clarity of this work. Vincenzo Salzano, Ruth Lazkoz, and Irene Sendra are supported by the Ministry of Economy and Competitiveness through research projects FIS2010-15492 and Consolider EPI CSD2010-00064, and by the Basque Government through research project GIU06/37. Support for Steven Rodney was provided by NASA through Hubble Fellowship grant \#HF51312.01 awarded by the Space Telescope Science Institute, which is operated by the Association of Universities for Research in Astronomy, Inc., for NASA, under contract NAS 5-26555.

\section{References}

Albrecht, A., Bernstein, G., Cahn, R., et al. 2006 [arXiv: astro-ph/0609591] Albrecht, A., Amendola, L., Bernstein, G., et al. 2009 [arXiv: 0901.0721] Amanullah, R., Lidman, C., Rubin, D., et al. 2010, ApJ, 716, 712

Astier, P., Guy, J., Regnault, N., et al. 2006, A\&A, 447, 31

Barai, P., Das, T. K., \& Wiita, P. J. 2004, ApJ, 613, L49

Bento, M. C., Bertolami, O., \& Sen, A. A. 2002, Phys. Rev. D, 66, 043507

Beutler, F., Blake, C., Colless, M., et al. 2011, MNRAS, 416, 3017

Blake, C., Kazin, E., Beutler, F., et al. 2011, MNRAS, 418, 1707

Carroll, S. M., Press, W. H., \& Turner, E. L. 1992, ARA\&A, 30, 499

Carroll, S. M., De Felice, A., Duvvuri, V., et al. 2005, Phys. Rev. D, 71, 063513

Chevallier, M., \& Polarski, D. 2001, Int. J. Mod. Phys. D., 10, 213

Choudhury, T. R., \& Padmanabhan, T. 2005, A\&A, 429, 807

Conley, A., Guy, J., Sullivan, M., et al. 2011, ApJS, 192, 1

Di Pietro, E., \& Claeskens, J. F. 2003, MNRAS, 341, 1299

Domínguez, I., Höflich, P., \& Straniero, O. 2001, ApJ, 557, 279

Dunkley, J., Bucher, M., Ferreira, P. G., Moodley, K., \& Skordis, C. 2005, MNRAS, 356, 925

Dvali, G. 2006, New J. Phys., 8, 326

Elgaroy, O., \& Multamäki, T. 2006, J. Cosmol. Astropart. P., 0609, 002

Freese, K., \& Lewis, M. 2002, Phys. Lett. B, 540, 1

Gong, Y. G., \& Zhang, Y. Z. 2005, Phys. Rev. D, 72, 043518

Gorini, V., Kamenshchik, A., \& Moschella, U. 2003, Phys. Rev. D, 67, 063509

Grogin, N. A., Kocevski, D. D., Faber, S. M., et al. 2011, ApJS, 197, 35

Guy, J., Astier, P., Baumont, S., et al. 2007, A\&A, 466, 11

Guy, J., Sullivan, M., Conley, A., et al. 2010, A\&A, 523, A7

Hicken, M., Challis, P., Jha, S., et al. 2009, ApJ, 700, 331
Huterer, D., \& Turner, M. S. 2001, Phys. Rev. D, 64, 123527

Ishak, M. 2007, Found. Phys., 37, 1470

Jassal, H. K., Bagla, J. S., \& Padmanabhan, T. 2005, MNRAS, 356, L11

Kessler, R., Becker, A. C., Cinabro, D., et al. 2009, ApJS, 185, 32

Kim, A. G., \& Linder, E. V. 2011, J. Cosmol. Astropart. P., 06, 020

Kim, A. G., Linder, E. V., Miquel, R., \& Mostek, N. 2004, MNRAS, 347, 909

Koekemoer, A. M., Faber, S. M., Ferguson, H. C., et al. 2011, ApJS, 197, 36

Komatsu, E., Smith, K. M., Dunkley, J., et al. 2011, ApJS, 192, 18

Kowalski, M., Rubin, D., Aldering, G., et al. 2008, ApJ, 686, 749

Lazkoz, R., Salzano, V., \& Sendra, I. 2010, Phys. Lett. B, 694, 198

Lee, S. 2005, Phys. Rev. D, 71, 123528

Liddle, A. R. 1998, An introduction to modern cosmology, Chichester (UK: Wiley)

Linder, E. V. 2003, Phys. Rev. Lett., 90, 091301

Linder, E. V. 2006, Phys. Rev. D, 73, 063010

Linder, E. V., \& Huterer, D. 2005, Phys. Rev. D, 72, 043509

Ma, J.-Z., \& Zhang, X. 2011, Phys. Lett. B, 699, 233

Maartens, R. 2007, J. Phys. Conf. Ser., 68, 012046

Nesseris, S., \& Perivolaropoulos, L. 2004, Phys. Rev. D, 70, 043531

Padmanabhan, T., \& Roy Choudhury, T. 2003, MNRAS, 344, 823

Percival, W. J., Reid, B. A., Eisenstein, D. J., et al. 2010, MNRAS, 401, 2148

Perlmutter, S., Aldering, G., Goldhaber, G., et al. 1999, ApJ, 517, 565

Postman, M., Coe, D., Benítez, N., et al. 2012, ApJS, 199, 25

Riess, A. G., \& Livio, M. 2006, ApJ, 648, 884

Riess, A. G., Filippenko, A. V., Challis, P., et al. 1998, ApJ, 116, 1009

Riess, A. G., Macri, L., Casertano, S., et al. 2011, ApJ, 730, 119

Sahni, V., \& Shtanov, Y. 2003, J. Cosmol. Astropart. P., 0311, 014

Sahni, V., \& Starobinski, A. 2000, Int. J. Mod. Phys. D, 9, 373

Sánchez, A. G., Baugh, C. M., Percival, W. J., et al. 2006, MNRAS, 366, 189

Seljak, U., Makarov, A., McDonald, P., et al. 2005, Phys. Rev. D, 71, 103515

Sotiriou, T. P., \& Faraoni, V. 2010, Rev. Mod. Phys., 82, 451

Sullivan, S., Sarkar, D., Joudaki, S., et al. 2008, Phys. Rev. Lett., 100, 241302

Sullivan, M., Guy, J., Conley, A., et al. 2011, ApJ, 737, 102S

Tegmark, M., Taylor, A. N., \& Heavens, A. F. 1997, ApJ, 480, 22

Tegmark, M., Strauss, M. A., Blanton, M. R., et al. 2004, Phys. Rev. D, 69 , 103501

Tsujikawa, S. 2010 [arXiv: 1004 . 1493]

Upadhye, A., Ishak, M., \& Steinhardt, P. J. 2005, Phys. Rev. D, 72, 063501

Wang, Y. 2008, Phys. Rev. D, 77, 123525

Wang, Y., Chuang, C.-H., \& Mukherjee, P. 2012, Phys. Rev. D, 85, 023517

Weinberg, D. H. 1972, Gravitation and Cosmology: Principles and Applications of the General Theory of Relativity (UK: J. Wiley)

Weinberg, D. H., Mortonson, M. J., Eisenstein, et al. 2012, Phys. Report, accepted [arXiv: 1201.2434$]$

Wetterich, C. 2004, Phys. Lett. B, 594, 17

Wood-Vasey, W. M., Miknaitis, G., Stubbs, C. W., et al. 2007, ApJ, 666, 694

Wolz, L., Kilbinger, M., Weller, J., \& Giannantonio, T. 2012, JCAP, 09, 009 